\title{
scripted
}

Volume 14, Issue 1, June 2017

\section{Book review: Medical Experimentation: Personal Integrity and Social Policy: New Edition}

\author{
Charles Fried (Franklin Miller and Alan Wertheimer, editors) \\ New York: Oxford University Press, 2016. 256 pages. \\ ISBN: $9780190602727 . £ 20$. \\ Reviewed by Edward S. Dove*

\section{(c) (1) $\odot$} \\ (c) 2017 Edward S. Dove \\ Licensed under a Creative Commons Attribution-NonCommercial- \\ NoDerivatives 4.0 International (CC BY-NC-ND 4.0) license
}

DOI: $10.2966 /$ scrip.140117.152

* PhD Candidate, School of Law, University of Edinburgh 
Medical Experimentation: Personal Integrity and Social Policy was written by Harvard Law School Professor Charles Fried more than 40 years ago and has been out of print until its republishing by Oxford University Press in 2016. The impetus to republish came about when Fried was approached by the editors, Franklin Miller and the late Alan Wertheimer, at a conference at Harvard Law School's Petrie-Flom Center in March 2013. The two had convinced Fried there was a need to republish the book in light of its ongoing strong resonance in contemporary medical issues, particularly randomised controlled trials (RCTs) the main subject of Fried's book. In his freshly written "Concluding Reflections", Fried explains that when he wrote Medical Experimentation in 1974, "the use of RCTs was relatively new as a means for evaluating both new and established pharmaceutical, surgical and other treatment modalities" (p. 220). Fried's academic work has long been in law and philosophy; the emerging "RCT offered a striking, intriguing and fresh context in which to consider and challenge a number of general ideas that occupied me at the time: consequentialism and rights, respect for persons, the concept of a life plan, problems of risk, the value of life, and what in the text I called the personalist argument" (pp. 220-221). This new edition contains an introduction from Miller and Wertheimer, a chapter from Harvard Law School Professors Glenn Cohen and James Greiner on whether and how one can ethically conduct RCTs in the legal setting (which I will not discuss here), and concluding reflections from Fried.

In this book, Fried provides a systematic, largely Kantian philosophical account of the ethics of RCTs. RCTs continue to be seen as the "gold standard" for evaluating the effectiveness of medical treatments. Across six chapters, Fried makes his case that the ethos of personal care is both an ideal interpersonal relationship between physicians and patients, and a set of rights owed to patients, grounded in respect for persons and operationalised in voluntary informed consent. After Fried presents the principles of American law applicable to 
medical experimentation in Chapter 2, he sketches his concept of personal care from Chapter 3 onwards. He argues that the most philosophically and socially challenging problems raised by human experimentation are those that relate "(1) to the conflict between the interests of the individual patient and his claim to the unreserved ministration of the physician, as opposed to the claims of more or less well defined and specified wider groups of persons, and (2) to the better formulation of the interest in good health" (p. 57). Fried addresses these questions separately and then brings the analyses together. His main concern lies with whether RCTs impose unjustified burdens on patients and the potential for RCTs to interfere with the physicians "duty of fidelity" to the patient. As Fried explains:

...the whole notion, so frequently repeated, that the RCT is peculiarly fair because it assigns persons to treatment categories at random rests on fallacy and confusion. It is fallacy to see some fairness in the fact that sickness may be an arbitrary, random event. And it is confusion to claim that the lottery is after all a fair one because in the RCT the treatment itself is distributed at random and the participants in the test do have equal chances of receiving one or the other treatment. For it is not this or that treatment which is the burden, the sacrifice to be justified, but rather the fact that all the participants in the trial are disadvantaged insofar as the care they receive is not chosen exclusively out of a concern for their individual well-being, but with regard to the success of the experimental design. (p. 75)

Fried makes a compelling case that a patient has a right to personal care, even at the cost of certain advantages to all. 
In Chapter 4, Fried argues that economic theory (in particular cost-benefit analysis, which in the 1970s was a burgeoning topic of discussion) is "inadequate to provide a complete account of what is best, what is right to do for the different persons involved in providing medical care" (pp. 91-92) precisely because economic analysis "has no theory of rights" and "what we are concerned with is precisely the question of rights in personal care" (p. 100). Economic theory is concerned with the weighing and balancing and set-off of interests against each other, whereas there are rights implicit in the concept of personal care and in respect to medical experimentation. For Fried, the goal of health care is to maintain the integrity of the human person, and as a correlation of this, the physician's prime and basic function is the preservation of the patient's "life capacities for the realization of a reasonable, realistic life plan" (p. 110). Fried makes the case that there are four rubrics or characteristics implicated by the fundamental concern about the integrity of the human person involved in medical care, namely lucidity, autonomy, fidelity and humanity.

Lucidity (Miller and Wertheimer suggest that in today's lingo, lucidity might be better phased as "transparency") means that a patient has a right to know all relevant details about the situation she finds herself in. Autonomy means that a patient has a right to be free from fraud in the relation of medical care, as well as from force and violence (e.g. treatment against one's will, except in rare and justified situations). Fidelity means that the patient must be able to rely on the physician's judgement and that the physician will be loyal to his patient's interests. Humanity means that over and above a right to be treated without deceit or violence, a person has a right to have her full human particularity taken into account by those who enter into relationships with her. In Chapter 5, Fried clarifies that these four rubrics "are not themselves the rights in the doctor-patient relationship but rather the characteristics which that 
relationship will manifest when the rights of doctors and patients are respected in it" (p. 148).

In his concluding Chapter 6, Fried brings his theory of personal care to bear on RCTs. Despite the strong claim that RCTs are the "gold standard" of biomedical research and the best hope of advancing medicine, RCTs may violate patients' rights for three reasons. First, research protocols may replace professional judgment as to what therapy is best for a particular patient because of the demands of the experimental design. Second, the practice of RCTs often involves deceit - the patient may believe that her doctor is determining her therapy solely on one basis, when there may be other factors involved about which the patient is not informed, and which might lead her to withdraw from the care of that particular doctor if she knew of them. Third, there are concerns about incomplete or deceptive disclosure of research results. This latter concern violates rights collected under the rubric of lucidity, while the former concerns violate rights associated with autonomy and fidelity. Thus for Fried:

The very vulnerability of the patient to his doctor creates expectations, expectations not just of truthfulness but of humane treatment. To disappoint those expectations is a wrong that goes beyond the actual harm that is done. For in disappointing those expectations the possibility of a system of trust is itself undermined. [...] The system of rights in personal care, applied to experimentation, entails the right to full disclosure, to complete candor, and the right not to be experimented upon against one's will, the right to choose one's own therapy with full awareness of the alternatives. (p. 173) 
This said, Fried does not believe RCTs are unethical per se; rather, his claim is that RCTs deviate from norms of personal care and it is crucial that they are designed to promote transparency and choice by patients to authorise or refuse research participation. In both instances, this is accomplished through the process of voluntary informed consent. Fried recognises that his concept of personal care may slow the progress of medicine, but, as he also submits, if "the confidence of patients in the profession and the attitude of respect in the profession for the patient is maintained, I believe it will have been well worth it" (p. 186).

I agree with Miller and Wertheimer that Fried's book should be seen as "a classic in the ethics of medical care and clinical research" (p. 1). Further, they are right to observe that in the current context of learning health care systems approaches and pragmatic RCTs, consent and RCTs take on new life - especially when seen in light of Fried's concept of personal care as defining the normative ethos of the doctor-patient relationship. While this book does not make for easy reading given its heavy philosophical bent and discussion of economic theory, it deserves nonetheless to be read by all those interested in contemporary medical issues. Bravo to Oxford University Press and to these editors for breathing new life into this celebrated contribution to the medical law and ethics literature. 